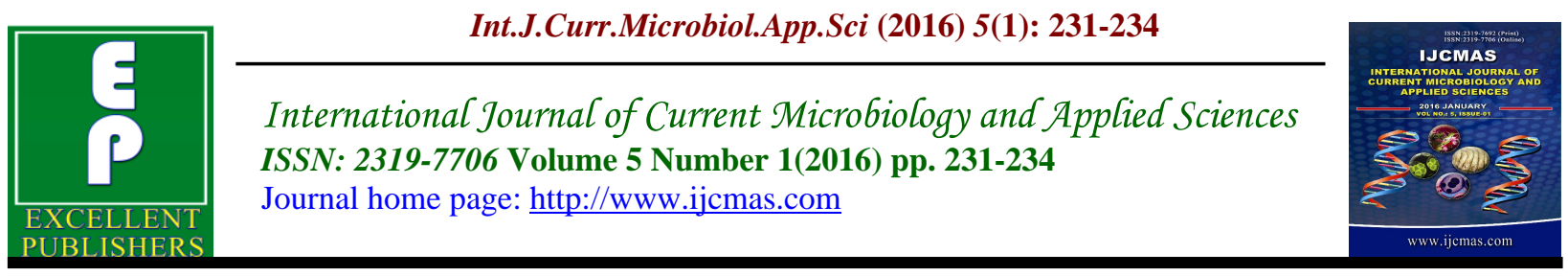

Review Article

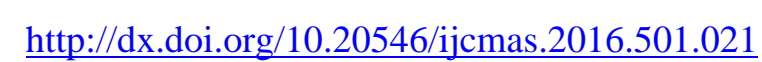

\title{
Bacteriophages as an Antibacterial Agent: A Promising Alternative
}

\author{
Kanupriya Dubey ${ }^{1}$, Sachin Chandraker ${ }^{1}$, Shweta Sao ${ }^{2}$, \\ Anjay Gupta ${ }^{1}$, and Sumit Kumar Dubey ${ }^{3}$ \\ ${ }^{1}$ Intermediate Reference Laboratory, Raipur, India \\ ${ }^{2}$ Dr. C. V. Raman University, Bilaspur C.G, India \\ ${ }^{3}$ NIT Raipur, India \\ *Corresponding author
}

\begin{abstract}
A B S T R A C T
Keywords

Bacteriophages,

Antibacterial

Agent,

pathogenic

bacteria

Article Info

Accepted:

12 December 2015

Available Online:

10 January 2016

Presently, the treatment of antibiotic resistant pathogenic bacteria has been extensively performed worldwide. The evolution of pathogenic bacteria against commercially available antimicrobial agents has become a critical problem in modern era. Phage therapy could be used as a potential alternative to antibiotics. Soviet Union used bacteriophages to treat infected soldiers during Second World War. Afterwards the Russian researchers started to work on the development of treatment via different Bacteriophages and further US researcher was actively involved on phase therapy and approached towards commercialization of phase therapy. Naturally occurring antibacterial agents are not able to overcome bacterial resistance, while Bacteriophages have a higher mutation and replication rate, so they can compete over the development of resistance of bacteria against antimicrobial agents.
\end{abstract}

\section{Introduction}

Bacteriophage belongs to viruses, and can be used to combat bacteria without a harmful effect on the human body. Bacteriophages have several characteristics that make them potential therapeutic agents. They are (i) highly specific and very effective in lysing targeted pathogenic bacteria, (ii) safe, and (iii) have ability to rapid modification to combat the emergence of newly arising bacterial threats (Fischbach M.A., 2009).
Discovery of penicillin (1928) channelize further breakthrough and the commercial production of antibiotics. Treatment of infectious disease by antibiotic therapy is most popular way. Antibiotics are manufactured about 100,000 tons annually worldwide. Application of antibiotics on pathogenic strains has become common practice. This makes pathogenic strains to develop more resistant against antibiotics and chemotherapeutic agents (Benjamine et. 
al., 2013). The emergence of pathogenic bacteria resistant to available antimicrobial agents has become a critical problem in modern medicine (Westergard, 2012) such as septicemia and ventilator-associated pneumonia, might no longer be successfully treated with antibiotics (National Center for Infectious Diseases, 2002). Drug resistant respiratory pathogens includes Streptococcus pneumoniae (Whitney et al., 2000; Grant 1997) and Mycobacterium tuberculosis (Nachega and Chaisson 2003). In 1917, d'Herelle tested phage in human patients, under the clinical supervision of Professor Victor-Henri Hutinel at the Hospital des Enfants- Malades in Paris. He demonstrated the safety level of phage by ingesting them inside the human body. The next day, he demonstrated their efficacy by administering phages to human body with severe dysentery. The patient's symptoms ceased after a single treatment, and he made a complete recovery. Dr. Herelle's anti-dysentery phage was then administered to three additional patients, all of whom began to recover within a day of treatments. (Setlow et al., 1992; Lorch, 1999).

\section{History}

The practice of phage therapy starts from decades ago. In 1896, Ernest Hankin revealed the antibacterial activity of bacteriophage against Vibrio cholerae. He worked on phases present in Ganga and Jamuna rivers located in India. A French microbiologist, Felix d.herelle observed bacteriophage phenomenon for first time in 1910. He was studied for the methods of controlling locusts in Mexico. While spread sample cultures on agar and after incubation period, round zones without growth were observed. Base on their observation they assumed the viral parasites were existed near zones. Six years later, he proposed the name bacteriophage (Duckworth, 1976). Later in1914 Frederick postulate the hypothesis that the agent may belongs to virus. Eliava Institute devoted to development of phage therapy, and suggested that an unidentified substance (not filterable, heat labile) was responsible for limiting the spread of cholera epidemics. Gamaleya, the Russian bacteriologist, observed a similar phenomenon while working with Bacillus subtilis 1914. Pasteur Institute in Paris and Eliava Institute in Tbilisi, Georgia were involved in extensive research and development in field the field of phase study. United States during the 1940s commercialize the concept of phage therapy, undertaken by the large pharmaceutical company, Eli Lilly. During the Second World War, the Soviet Union applied phage therapy to treat soldiers. Soldiers were infected with different bacterial diseases e.g. dysentery and gangrene. Russian researchers continued to development and evaluation of their treatment methods to publish their research. A summary of these publications was published in "A Literature Review of the Practical Application of Bacteriophage Research". Major breakthrough occurred in 1994; when Soothill demonstrated the administration of phages in an animal model that was improve the success of skin grafts by reducing the Pseudomonas aeruginosa infection.

\section{Advantages of Phage Therapy}

An advantage of Phage treatment offers a potential substitute over conventional approach (antibiotic treatments). Bacteriophages are very specific, targeting only one or a few strains of bacteria. Traditional antibiotics have more wideranging effect that killed both harmful bacteria and beneficial bacteria. But specificity of bacteriophages might reduce the chance of harmful effect on beneficial bacterial strains inside human body. 
Bacterial strains of Enterobacter sakazakii (Kim et al., 2007) and Listeria Monocytogenes (Setlow et al., 1992) was studied against the infectious pathogen resistance. Effective dose of phase can able to completely eradicate the target organisms or an alternatively phages can be applied or mixed directly onto or into the food product (Ho, 2001).

\section{Limited Impact}

Bacteriophages are self-replicating and selflimiting based on the availibity of target bacterial spp inside the human body. Bacteriophages are replicate exponentially when specific (Target) bacteria are available in abundance, and decreasing of specific bacterial handiness limited the number of phages or get decay. As time elapsed the Bacteriophases are eliminated from the patient. Depending on the form of application, a single dose may be sufficient.

\section{Limited Resistance Development}

Bacteria will certainly develop resistance over phase therapy. However, phages have a higher mutation and replication rate, so they can compete to the adaptation of the bacteria. Thus the development of bacterial resistance to the bacteriophages is limited. No serious side effects have been reported on bacteriophages, towards human health as well as environment (Lorch,1999).

\section{Obstacles of Phage Therapy}

Due to the higher specificity of Bacteriophase over bacterial strain, needs to makes different cocktails for the same types of treatment (of infection or disease). Because the bacterial strains or components are differ from region to region or even may vary with person to person. Another problem associated with phase therapy is based on the fact that protein-based pharmaceuticals can stimulate immune systems. Antibiotics that lyse bacteria will release bacterial toxins in situ that infects cells and tissues of the body. Additionally, due to the specificity of individual phages, a mixture of phages is often applied for a high chance of success. This means that 'banks' containing many different phages must be kept and regularly updated with new phages, which is a tedious job.( Keen., 2012). Protein-based drugs, chemical antibiotics, and whole vaccines have previously been approved for use. Therefore phage-based pharmaceuticals could possess an equal chance of getting approved with similar sets of attributes (Loc-Carrillo Catherine., 2011). The greatest obstacle to introducing bacteriophages in our healthcare is that the pharmaceutical companies show little enthusiasm, since phages are difficult to patent.

In conclusion, bacteriophages are proteinbased, biological agents that can potentially interact with the body's immune system. It can actively replicate, and evolve after administration. Its higher replication rate has been also beneficial at the commercial production of phages. In addition, a large number of publications, some of which are reviewed in this mini review, suggest that phages may be effective therapeutic agents in selected clinical settings. Moreover, phages can be used as vehicles for DNA and protein based vaccines, for the detection of pathogenic bacterial strains.

\section{References}

Adhya S, Merril C (2006) The road to phage therapy. Nature 443: 754-755.

Benjamin K.C., Stephan T., Abedon., Catherine Loc-Carillo., "Phage cocktail and future of phage therapy," Future Microbiology, vol.8 No.6, pp.769-783, 
June.

Centers for Disease Control (2002), Antimicrobial resistance: a growing threat to public health. Atlanta: Division of Healthcare Quality Promotion; National Center for Infectious Diseases

Duckworth DH (1976) Who discovered bacteriophage? Bacteriol Rev 40: 793802.

Fischbach M.A., Walsh C.T., "Antibiotics for emerging pathogens". Science vol. 325 (5944) pp, 1089-1093, April 2009.

Frank R.D., Henry F.Chambers., "Reemergence of antibiotic resistant staphylococcus aureus in the genomic era." The journal of clinical Investigation,vol-9,pp.24642474,September 2007.

Grant GR, Lederman JA, Brandstetter RD (1997) T.G. Heaton, tuberculosis, and artificial pneumothorax: once again, back to the future? Chest 112: 7-8.

Herelle F.d., "An invisible microbe that is antagonistic to the dysentery bacillus". Virology ,vol.165,pp.373-375,1917

Ho K (2001) Bacteriophage therapy for bacterial infections: rekindling a memory. Perspect Biol Med 44: 1-16.

Keen E.C., "Phage Therapy: Concept to Cure". Frontiers in Microbiology vol. 3 .pp.1-3, July, 2012.

Kim KP, Klumpp J, Loessner MJ (2007) Enterobacter sakazakii bacteriophages can prevent bacterial rowth in reconstituted infant formula. Int $\mathrm{J}$ Food Microbiol 115: 195- 203.
Loc-Carrillo. Catherine, Stephen T.Abendon., "Pros and cons of phage therapy." Bacteriophage, vol.-1,pp.111114,March-April 2011.

Lorch Antje., "Bacteriophages: An alternative to antibiotics" Biotechnology and Development monitor, No.39, pp.14-17, September 1999.

Matsuzaki S., Rashel M., Uchiyama J., et.al., " Bacteriophage therapy: a revitalized therapy against bacterial infectious diseases". Journal of. Infection and Chemotherapy vol.5, pp,211-219 October 2005.

Nachega JB, Chaisson RE (2003) Tuberculosis drug resistance: a global threat. Clin Infect Dis 36: 24-30.

Setlow J, Randesi M, Adams J, Setlow B, Setlow P (1992) Mutation and killing of Escherichia coli expressing a cloned Bacillus subtilis gene whose product alters DNA conformation. J Bacteriol 174: 2943-2950.

Twort F.W., "An investigation on the nature of ultra-microscopic viruses" Lancet vol 186,pp.1241-1243 December 1915.

Westergard Per., "Sustainability" October 2012.

Whitney CG, Farley MM, Hadler J, Harrison LH, Lexau C, Reingold A (2000) Increasing prevalence of multidrugresistant Streptococcus pneumoniae in the United States. N Engl J Med 343: 1917-1924.

\section{How to cite this article:}

Kanupriya Dubey, Sachin Chandraker, Shweta Sao, Anjay Gupta, and Sumit Kumar Dubey. 2016. Bacteriophages as an Antibacterial Agent: A Promising Alternative. Int.J.Curr.Microbiol.App.Sci. 5(1): 231-234. ihttp://dx.doi.org/10.20̄5 $\overline{4} 6 \overline{6} / \mathrm{ijcmas} .2016 .501 .02 \overline{1}$ 